\title{
The future of accelerator-based physics after LHC
}

\author{
Franco Bedeschi* \\ INFN, Sezione di Pisa \\ E-mail: franco.bedeschi@pi.infn.it
}

At the apex of its success, after the Higgs boson discovery, high energy particle physics has little guidance on how to best invest for its future. Nonetheless important decisions must be made in relatively short timescale to secure a continuation of this field of research after the end of the Large Hadron Collider high luminosity experiments. We discuss physics motivations and technology issues that can provide indications for the choice of a major future research facility among the many proposed so far.

VII Workshop italiano sulla fisica pp a LHC

16-18 Maggio 2016

Pisa, Italy

${ }^{*}$ Speaker. 


\section{Introduction}

The present highest energy accelerator, the Large Hadron Collider (LHC), is extremely successful in exploring the Standard Model (SM) of particle physics at levels of sensitivity never achieved until now. The discovery and then the study of the Higgs boson is a major achievement in the field of High Energy Physics (HEP). The LHC will continue to increase its luminosity in the years to come and is foreseen to run until approximately 2035. The are several new accelerators that could be in operation on that timescale or later, however at the moment there is no clear plan on what the new big machine will be.

For $e^{+} e^{-}$collisions the main options are: the International Linear Collider (ILC) in Japan, a linear collider with $500 \mathrm{GeV}$ center of mass energy (extendable to $1000 \mathrm{GeV}$ ), the Compact LInear Collider (CLIC) at CERN, another linear collider with energy up to $3 \mathrm{TeV}$, the Future Circular Collider (FCC-ee) at CERN, a circular collider inside a new $\sim 100 \mathrm{~km}$ tunnel with energies up to $350 \mathrm{GeV}$, and the Chinese electron-positron Collider (CepC) in China, a circular collider inside a $54 \mathrm{~km}$ tunnel and energy up to $250 \mathrm{GeV}$.

Both the FCC-ee and the CepC options foresee the use of the same tunnel for a proton-proton machine of very high energy called FCC-hh and SppC respectively. If adequate magnets will be available, an energy as high as $100 \mathrm{TeV}$ could be reached.

Additional options assume that some critical technologies can become at some point mature and available on an industrial scale. This of course pushes the time frame for the implementation of such machines further out in the future. Important examples are Plasma Wake Field Acceleration (PWFA) and muon colliders.

In the following sections we discuss where physics interests lead, analyze in more detail all the options mentioned here and then report our conclusions.

\section{Current status}

The SM is providing more than ever an excellent description of the experimental data. This is true also for the most recent results obtained at the highest available energy provided by the LHC. The discovery of the Higgs boson and preliminary measurements of its properties, like mass, crosssection and quantum numbers, are confirming, although with modest accuracy, the SM framework and its predictions.

Additional impressive confirmations come from measurements of quantities which are sensitive to radiative corrections such as the $\mathrm{W}$ mass, where these corrections depend on the measured masses of the top quark and the Higgs boson, or the branching ratios of very suppressed processes such as the decay of neutral B mesons into a pair of opposite sign muons. Given the potential presence of new particles in virtual loops, the increased accuracy of these measurements sets stronger limits on physics beyond the SM.

Indeed also the direct searches do not show any clear indication for new physics in spite of the massive efforts made by the LHC collaborations, thus confirming a scenario where the mass scale of new physics is being pushed toward energies much higher than originally expected by many models. 
This solid scenario is broken at times by small discrepancies that usually fade away with additional data. Foremost, for the recent excitement it has generated, is the unexpected appearance of a di-photon invariant mass peak around $750 \mathrm{GeV}$ with the statistical significance of a few standard deviations. We will know soon, with additional data, if it is really new physics or just a fluctuation. In case it is confirmed, it would be the start of a new era in particle physics and very clear indications for the next accelerator to build would come from this. If not, then we shall have to follow the directions suggested in the next section.

\section{Directions}

The conclusion of the preceding section is in contrast with the fact that the SM fails to describe some very important phenomena like gravitation, dark matter or baryonic asymmetry of the universe just to name a few. A more complete theory must therefore exist, but we have only lower bounds on its energy scale and do not know its typical signatures.

At a recent ECFA meeting a well known theorist concluded that given the situation "there is currently no experiment or facility, existing or conceivable, in the lab or in space, accelerator or non-accelerator driven, that can guarantee discoveries beyond the SM, and answers to the big questions in the field." [1]. During that same meeting, however, some very sensible guidelines for the evaluation of future facilities have emerged:

1. guaranteed important deliverables;

2. a wide exploration potential, targeting broad and well justified new physics scenarios;

3. potential to provide conclusive answers to relevant questions.

We add to that some additional practical criteria:

a) the time scale for the construction must be consistent with the evolution of the required technologies,

b) the expected construction and operation costs must be within an acceptable range.

We now explore in more detail how new machines can satisfy the proposed guidelines. Important deliverables come from high precision measurements, beyond what is achievable at the LHC, even with its high luminosity upgrade [2][3][10]. A special case is the detailed study of the properties the Higgs boson, which can provide crucial insights on how the SM should be extended. Examples for effects of Supersymmetry or Higgs boson compositness [10] are shown in fig. 1. Deviations of the Higgs couplings relative to SM predictions range from few percent to sub-percent for new physics scales between 1 and $10 \mathrm{TeV}$. A new generation of high luminosity electron-positron colliders running above the Higgs $+\mathrm{Z}$ boson associate production threshold could reach experimental accuracies much higher than LHC. Comparisons of the reach of LHC, CepC and ILC are shown in fig. 2 [3]. Higher luminosity and precision is foreseen for the FCC-ee, where most couplings could be measured with sub-percent relative errors.

Measurements of the Higgs self-coupling allow to study the shape of the Higgs potential, which is seriously constrained by its mass within the SM. If the experimental error could be reduced 

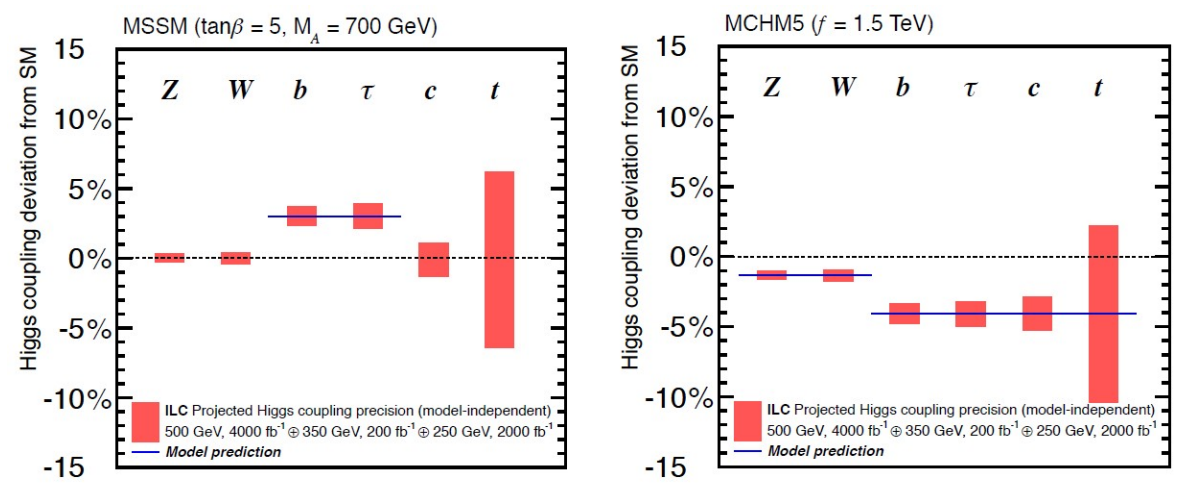

Figure 1: Examples of how new physics models can change the pattern of Higgs boson couplings [4].
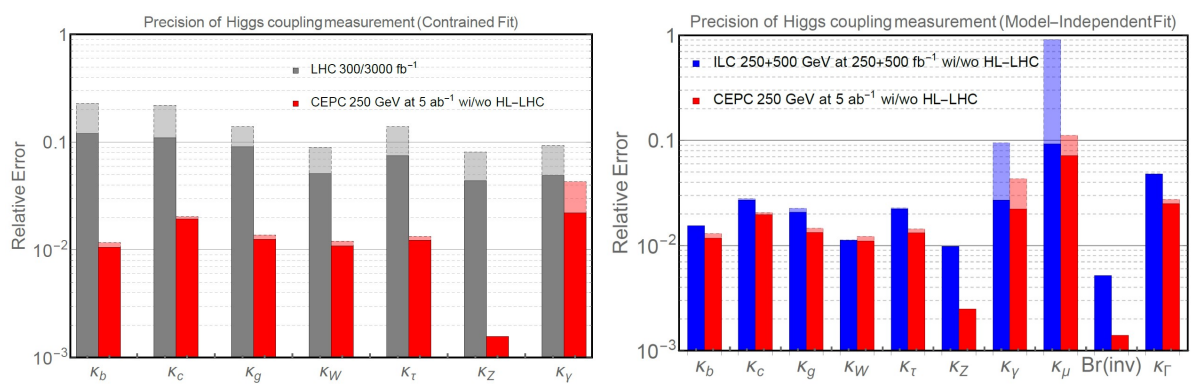

Figure 2: Comparison of relative accuracy in Higgs boson coupling measurements achievable with different accelerators [3].

below the $10 \%$ level we would be sensitive to new physics effects [5]. This is an incredibly difficult measurement and needs a high Higgs boson production cross-section that can be reached only at lepton colliders in the $\mathrm{TeV}$ region or with proton-proton colliders near $100 \mathrm{TeV}$.

Additional precision measurements in the electro-weak sector can improve significantly previous LEP results and provide guidelines for new theories through an Effective Field Theory analysis of potential deviations from the SM [6][2]. The extreme luminosity foreseen for future $e^{+} e^{-}$colliders, orders of magnitude larger than LEP, could make this line of research very fruitful.

The exploration potential of future accelerators depends somewhat on the class of models we intend to explore. A good reference is SUSY since it is one of the most widely studied scenarios of new physics. The sensitivity of LHC with $3000 \mathrm{fb}^{-1}$ ranges from masses of $\sim 1.5 \mathrm{TeV}$ in the electro-weak sector to $\sim 3 \mathrm{TeV}$ for gluino production. Sensitivity improvements of about $30 \%$ are expected if the LHC energy is raised to $25 \mathrm{TeV}$, while factors of 5 or more could be reached with a $100 \mathrm{TeV}$ pp collider. We note that lepton colliders with energies of a few $\mathrm{TeV}$ can have comparable reach in the electro-weak sector.

Finally we give an example of a potential conclusive answer to a major question of the field. A study based on a simplified model where Dark Matter couples to ordinary fermions via a mediator [8][7], shows that the parameter space allowed by the recent Plank results [9] could be covered by a $100 \mathrm{TeV}$ pp machine. 
In conclusion there are several compelling physics motivations to build new accelerators. In the following section we discuss what could actually be done.

\section{Options}

Several options for new machines have been proposed and some of them have already been on the table for quite some time. Some of them are based on well established technologies and require only funding to be realized, while other projects either need more $R \& D$ or even the demonstration of novel technical approaches.

\subsection{Accelerators based on known technology}

After more than 20 years of developments in the superconducting RF cavity technology the International Linear Collider (ILC) is a very mature project [10]. A full TDR has been released in 2013 and after that the project has gone through several successful reviews. A site where it could be built has also been selected in Japan. Negotiations between governments are in progress to establish if a realistic sharing of the costs can be found. This process will take at least another two or three years.

While the ILC is planned to run $e^{+} e^{-}$collisions mostly at a center of mass energy of 500 $\mathrm{GeV}$ and can be extended up to $1000 \mathrm{GeV}$, large circular $e^{+} e^{-}$colliders could provide substantially higher luminosity in a lower energy range that could however cover from $\mathrm{Z}$ production to $\mathrm{H} \mathrm{Z}$ associated production and possibly even top pair production. CERN launched in 2014 the idea of a future circular collider ( FCC) to be placed in an approximately $100 \mathrm{~km}$ long tunnel in the Geneva area [11]. The ultimate goal of this machine would be proton-proton collisions at $100 \mathrm{TeV}$, but an intermediate stage as $e^{+} e^{-}$collider is also considered as a potential intermediate step. A CDR is planned for the end of 2018 to provide input to the next European Strategy update. A similar machine to be built in China [3] was also proposed at approximately the same time [12]. In this case a Pre-CDR [3] was completed last year and a full CDR is planned for mid-2017.

We note that while all these proposed machines, at least in their $e^{+} e^{-}$version, are based on known technologies, they are nonetheless pushing the luminosity to rather extreme levels: two to five orders of magnitude higher than the maximum luminosity reached at LEP as shown in fig. 3. Much attention is given to the power consumption and the associated operating costs. The luminosities shown in the figure appear to be consistent with acceptable power requirements.

\subsection{Accelerators needing more $R \& D$}

The final energy of future proton-proton colliders will depend critically on the availability of a new generation of magnets capable of much higher fields. Indeed there are many practical limitations on the tunnel size, so magnets become the main issue. Superconductor cables based on $\mathrm{Nb}_{3} \mathrm{Sn}$ have shown critical currents 2-3 times higher than that of ordinary NbTi cables. They are however very difficult to work with since they are very brittle and require the development of complex construction techniques. The higher fields inside these magnets generate forces several times larger than in NbTi magnets enhancing coil stability problems. Significant R\&D on $\mathrm{Nb}_{3} \mathrm{Sn}$ magnet technology has been started since many years mostly at CERN and Fermilab, and a few magnets of this type are foreseen for the low beta triplets of the high luminosity upgrade of LHC 


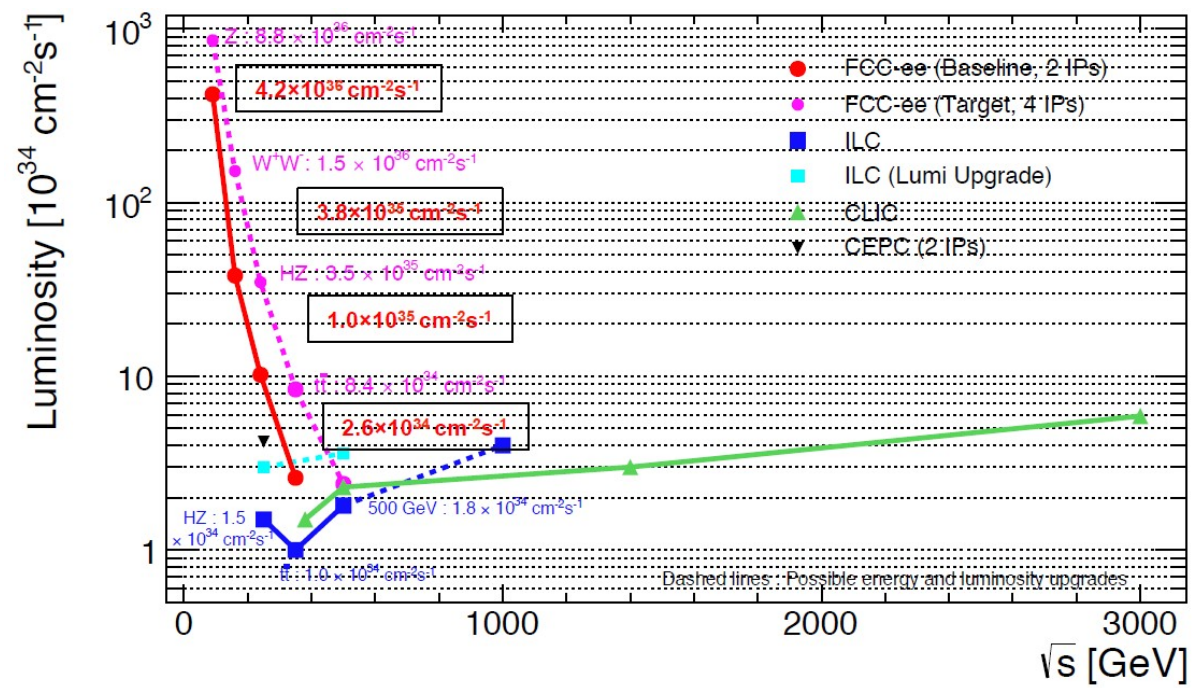

Figure 3: Comparison of luminosity planned for various $e^{+} e^{-}$colliders versus the operating energy [13].

due to start in 2025. Still we are far from having industrial production capability. CERN has setup an ambitious R\&D program for FCC-hh that could lead to the first dipole prototypes around the mid-2020's. Additional increase of the magnetic field could be obtained with the use of High Temperature Superconductors (HTS). While the LHC dipoles are operated at a field value of 8 Tesla, 16 Tesla are planned using the $\mathrm{Nb}_{3} \mathrm{Sn}$ technology and 20 Tesla could be possible with the HTS inserts.

Additional R\&D is also needed for the CERN multi TeV linear $e^{+} e^{-}$collider, CLIC [14]. This machine is based on the novel concept of using a high intensity low energy beam as the source of RF power rather then the usual klystrons. The high frequency accelerating structures operate at 12 $\mathrm{GHz}$ and room temperature reaching an accelerating field of $100 \mathrm{MV} / \mathrm{m}$. While several aspects of this very complex machine have been demonstrated there are still many challenges to deal with, in particular the operating power and the RF breakdown issues. A plan for a staged implementation is being prepared for consideration in the next European Strategy update[15].

\subsection{New accelerator concepts}

Muon colliders could provide the most efficient way to collide leptons in the several $\mathrm{TeV}$ region; indeed studies show [16] that above center of mass energy of $\sim 1.5 \mathrm{TeV}$ a muon collider is superior to all other lepton colliders both in terms of luminosity and of wall power consumption. Muons however are hard to produce and decay rapidly, thus making a muon collider an incredibly difficult machine. Two basic approaches have been studied so far: the first one relies on producing a very large number of muons by means of a proton beam with a power of several MW and then cooling them very rapidly by means of a method called "ionization cooling", the second approach consists in producing muon pairs by colliding a $45 \mathrm{GeV}$ positron beam on a target [17], since muon pairs are created roughly at rest in their center of mass frame there would be no need for significant cooling. Both techniques are incredibly challenging, but certainly worth pursuing given the many 
advantages that such a machine could have. It is worth noting that future high energy $e^{+} e^{-}$circular colliders could provide the intense $45 \mathrm{GeV}$ positron beam needed for muon generation.

Even more exotic is the relatively new idea to accelerate particles with the strong electric field obtained by generating waves in a plasma, where fields as high as $100 \mathrm{GeV} / \mathrm{m}$ can be reached. Plasma waves can be excited either with a strong laser pulse or with a particle beam. Much progress has been made in this area of research over the last years. Recent breakthroughs are the acceleration of positrons in a plasma [18] and the successful synchronization of two laser plasma accelerator stages [19].

It will take still some time before plasma wake field acceleration can provide high energy at the high luminosity needed by HEP experiments, but speculations are already being made on how one could significantly enhance the ILC energy [20] up to $10 \mathrm{TeV}$ by replacing the RF cavities with plasma cells excited by the electron beam.

\section{Conclusions}

The current state of HEP is quite complex with no clear indication of where the next major discovery will be found. There are however sensible guidelines that indicate quite clearly what is important to study in this field of research and which new facilities would be necessary. The actual time sequence of these new facilities will depend strongly on the speed at which new technologies develop, on their cost and, also, on geopolitical factors.

By the mid-2030's the LHC will have concluded its usefulness and it is critical that a new machine starts operation before that happens. An $e^{+} e^{-}$collider is the most likely next step: its physics goals are very clear; it needs well established technologies; the costs are high but manageable and in any case comparable to those of LHC. It is probably the only machine that could be built and become operational by the beginning of the 2030's. In a few years the situation in Asia for CepC and ILC should become clearer. At approximately the same time Europe will update its strategy plan: we will know better then about the real time scale for FCC and about potential cooperation with projects in Japan and China. In the meantime it is important to keep investing on accelerator related technologies and to get ready for the next big machine.

\section{References}

[1] M. L. Mangano, "Physics Highlights of Future pp Colliders", $98^{\text {th }}$ Plenary ECFA Meeting, CERN, 19-20 November 2015

[2] A. Andreazza et al., "What Next: White Paper of CSN1 - Proposal for a long term strategy for accelerator based experiments", Frascati Phys. Ser. 60 (2015) pp. 1-291, Editors: F. Bedeschi, R. Tenchini, J. Walsh, ISBN 978-88-864-0999-5

[3] CEPC-SPPC Study Group, "CEPC-SPPC Preliminary Conceptual Design Report. 1. Physics and Detector," IHEP-CEPC-DR-2015-01, IHEP-TH-2015-01, HEP-EP-2015-01.

http://inspirehep.net/record/1395734/files/main_preCDR.pdf

CEPC-SPPC Study Group, “CEPC-SPPC Preliminary Conceptual Design Report. 2. Accelerator," IHEP-CEPC-DR-2015-01, IHEP-AC-2015-01.

http://inspirehep.net/record/1395736/files/Pre-CDR_final_20150317.pdf 
[4] K. Fujii, et al., "Physics Case for the International Linear Collider", ILC-NOTE-2015-067, DESY 15-094, KEK Preprint 2015-16, LAL 15-188, MPP-2015-120, SLAC-PUB-16302, arXiv:1506.05992 [hep-ex]

[5] R. S. Gupta et al., "How well do we need to measure the Higgs boson mass and self-coupling?", Phys. Rev. D 88, 055024

[6] J. Ellis, T. You, "Sensitivities of Prospective Future $e^{+} e^{-}$Colliders to Decoupled New Physics", JHEP 03 (2016) 089, arXiv:1510.04561v1 [hep-ph]

[7] M. McCullough, "BSM Physics at FCC-hh", FCC week, Rome, Italy, April $12^{\text {th }} 2016$, https://indico.cern.ch/event/438866/contributions/1085049/attachments/1256400/1854877/FCCWeek.pdf

[8] P. Harris, V. V. Khoze, M. Spannowsky and C. Williams, "Closing up on Dark Sectors at Colliders: from 14 to 100 TeV,” Phys. Rev. D 93, no. 5, 054030 (2016).

[9] P. A. R. Ade et al. [Planck Collaboration], "Planck 2015 results. XIII. Cosmological parameters," arXiv:1502.01589 [astro-ph.CO].

[10] T. Behnke et al., "The International Linear Collider Technical Design Report - Volume 1: Executive Summary," arXiv:1306.6327 [physics.acc-ph],

H. Baer et al., "The International Linear Collider Technical Design Report - Volume 2: Physics," arXiv:1306.6352 [hep-ph],

C. Adolphsen et al., "The International Linear Collider Technical Design Report - Volume 3.I:

Accelerator R\&D in the Technical Design Phase,” arXiv:1306.6353 [physics.acc-ph],

C. Adolphsen et al., "The International Linear Collider Technical Design Report - Volume 3.II: Accelerator Baseline Design,” arXiv:1306.6328 [physics.acc-ph],

T. Behnke et al., "The International Linear Collider Technical Design Report - Volume 4: Detectors," arXiv:1306.6329 [physics.ins-det].

[11] M. Benedikt, F. Zimmermann, "Future Circular Collider (FCC) Study", Future Circular Collider Study Kickoff Meeting, University of Geneva, Geneva, Switzerland, February 2014.

[12] Y. F. Wang, "Welcome and Introduction", International Workshop on Future High Energy Circular Colliders, IHEP, Beijing, China, December 2013.

[13] Courtesy of Patrick Janot, CERN.

[14] M. Aicheler et al., "CLIC Conceptual Design Report", SLAC-R-985, KEK Report 2012-1, PSI-12-01, JAI-2012-001, CERN-2012-007.

[15] P. Burrows, "CLIC Accelerator: status, plans and outlook", CLIC Workshop 2016, CERN, January 2016.

[16] J. P. Delahaye,"Unique Properties of Muon Beams", Discussion of the scientific potential of muon beams, CERN, November 2015.

[17] M. Antonelli, E. Bagli, M. Biagini, M. Boscolo, G. Cavoto, P. Raimondi and A. Variola, "Very Low Emittance Muon Beam using Positron Beam on Target," doi:10.18429/JACoW-IPAC2016-TUPMY001, M. Antonelli, M. Boscolo, R. Di Nardo and P. Raimondi, "Novel proposal for a low emittance muon beam using positron beam on target,” Nucl. Instrum. Meth. A 807, 101 (2016).

[18] S. Corde et al., "Multi-gigaelectronvolt acceleration of positrons in a self-loaded plasma wakefield," Nature 524, no. 7566, 442 (2015). 
[19] S. Steinke et al., "Multistage coupling of independent laser-plasma accelerators," Nature 530, no. 7589, 190 (2016).

[20] J. P. Delahaye, "BEAM DRIVEN PLASMA-WAKEFIELD LINEAR COLLIDER FROM HIGGS FACTORY TO MULTI-TEV", THPRI013, Proceedings of IPAC2014, Dresden, Germany. 